\title{
Theoretical and experimental aspects of the controlled movement of solenoid actuators by means of the voltage static converter with logic gates
}

\author{
Flaviu-Nicolae Kesucz \\ Department of Engineering and \\ Management of Technology \\ Technical University of ClujNapoca, \\ North University Center \\ Baia Mare, Romania \\ flaviu.kesucz@cunbm.utcluj.ro
}

\begin{abstract}
The purpose of the scientific research in this paper is to control the movement of electromechanical actuators, through a new voltage convertor. The optimization of the system consists in finding new solutions to control the movement of electromechanical actuators in the gas valve, used in thermal power plants, which can be operated by static converters - with logic gates.
\end{abstract}

Keywords-actuators, control, converters, electromechanical, logic gates, motion, Proteus.

\section{INTRODUCTION}

Energy-impacted products whose use has an influence on energy consumption have a high share of energy consumption in the European Union. Known as the Eco Design Directive, ErP (Energy related Products) provides for the reduction of energy consumption as well as the energy labeling of energy consuming products by 2020. [2], [15]

Experimental research presents one of the main ways of approaching the problems of scientific, fundamental, applied investigation. It has a significant share in scientific research and is one of the main ways to address investigative issues. [5]

The purpose of scientific research in this paper is to improve the operation of thermal power plants, by optimizing the movement of electromechanical actuators, which are integrated into the composition of gas valves, in terms of adaptation to the requirements of comfort, efficiency and maintenance. The optimization of the system consists in finding new solutions to control the movement, operation of electromechanical actuators in the gas valve, used in thermal power plants, to be operated by static converters - AC voltage converter. The development of scientific knowledge in the field of intelligent manufacturing systems is achieved through the new approach to optimized motion control of electromechanical actuators, used in thermal power plants and not only, by creating new command and control modules, opens new horizons in design research, their realization and use, which should meet the requirements in the field of mechanical-mechatronic engineering. As this scientific research project presents the improvement of the operation of thermal power plants, respectively of gas valves by optimizing the movement of electromechanical actuators, it will be achieved through a new experimental AC voltage converter with logic gates.

\subsection{General information on static converters}

Static converters (CS) are equipment whose power part contains semiconductor power elements. Uncontrolled static converters are built with uncontrolled semiconductor devices (diodes) and also convert electricity into electricity, changing its parameters, without allowing the regulation of the average power transmitted to the load. [10]

Alternating-alternating converters, also called alternating voltage converters, are complex static equipment interspersed between the power source and the receiver, having the role of modifying the parameters of the energy supplied by the source, taking into account the requirements imposed by the receiver. [8]

In Figure 1, is shown the energy flow is oriented from the alternating current side of the input to the alternating current side of the output. The output frequency is the same as the input frequency, but the value of the output voltage can be changed. [10]

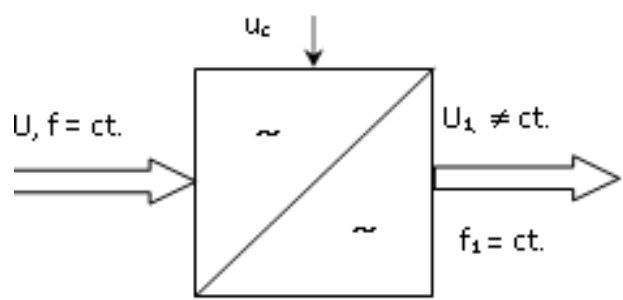

Figure1 Static converters a.c. or AC voltage converter

Controlled static converters which are built with controlled semiconductor elements and which, in addition to converting electricity, allow the control of the average power transmitted to the load. In the energy flow, the static converter is placed between the primary power generator (GP) which supplies electricity with constant parameters (voltage amplitude, frequency) and the load (S) which is a consumer of electricity. In the Figure 2 shows the place of static converters in the energy flow. 


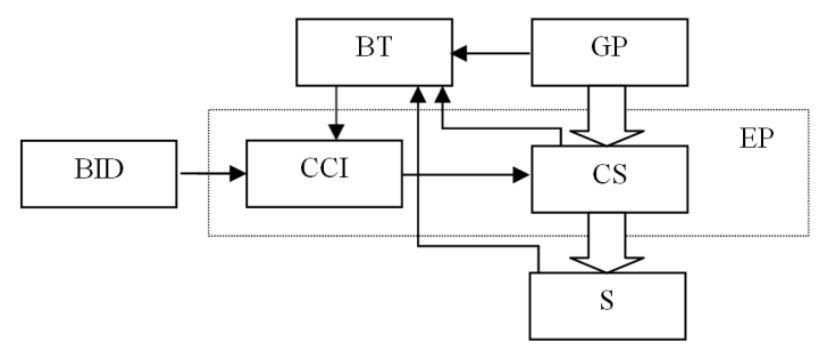

Figure 2 Explanatory regarding the place of CS in the energy flow

The following blocks are for the information flow:

BID - data entry block, has the role of entering the initial data for the command and consists of: keys, buttons, keyboard, information readers;

CCI - closed circuit control block, develops one or more control signals based on an established control strategy and quantities that characterize the operation of the entire system (current intensity, electrical power, rotational speed);

CS - the static converter, together with the closed circuit control block, forms the field of power electronics (EP);

\section{$\mathbf{S}$ - represents the load, is a consumer of electricity;}

GP - represents the primary source of electricity, usually providing electricity of alternating current or direct current with constant parameters (battery, mains, transformer);

BT - the block of transducers has the role of converting the quantities collected from the system into electrical quantities (voltages or currents) compatible in form and value with the CCI inputs. CCI together with CS form the object of power electronics. In modern systems, the CCI functions are assigned to a microprocessor or a dedicated computer. [10]

Static converters contain two parts and can be seen in Figure 3.

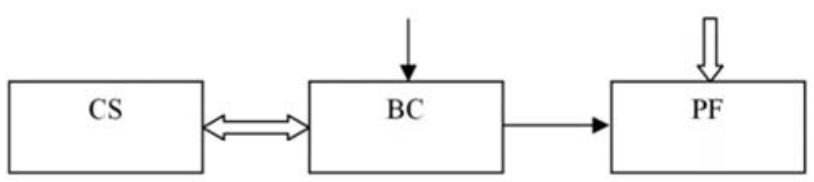

Figure 3 Component parts of static converters

where:

PF - the force part contains semiconductor controlled or semi-controlled power elements and their related protection blocks;

BC - control block, is made with elements specific to weak currents and has the role of generating the control signals necessary for power semiconductors and to distribute them.

Static converters are complex static equipment interspersed between the energy source and the receiver, having the role of modifying the parameters of the energy fumigated by the source (value, shape, frequency, voltage) taking into account the requirements imposed by the receiver. The converters can also be mounted between two power sources to make their simultaneous operation possible. The static converter acts as a receiver in terms of power source and as a power source in terms of load. [8]
The converters can also be mounted between two power sources, to make possible their simultaneous operation. The static converter acts as a receiver in terms of power source and as a power source in terms of load.

The power part of the receiver is made with controllable power semiconductor devices (thyristors, transistors). These devices, operating in switching mode, have the role of switches, so it results in a permanent mode consisting of a periodic succession of transient modes.

The successive closing and opening of these switches is done according to a logistics imposed by the operating principle of the converter. This logic is provided by the electronic control diagram. All converters therefore contain a part of power (force) and a part of control. Converters ensure the conversion of significant amounts of energy. It requires that their main sizing criterion be efficiency. This causes differences in power and signal electronics, where the main goal is to obtain a reliable output signal. Figure 4 shows energy conversions.

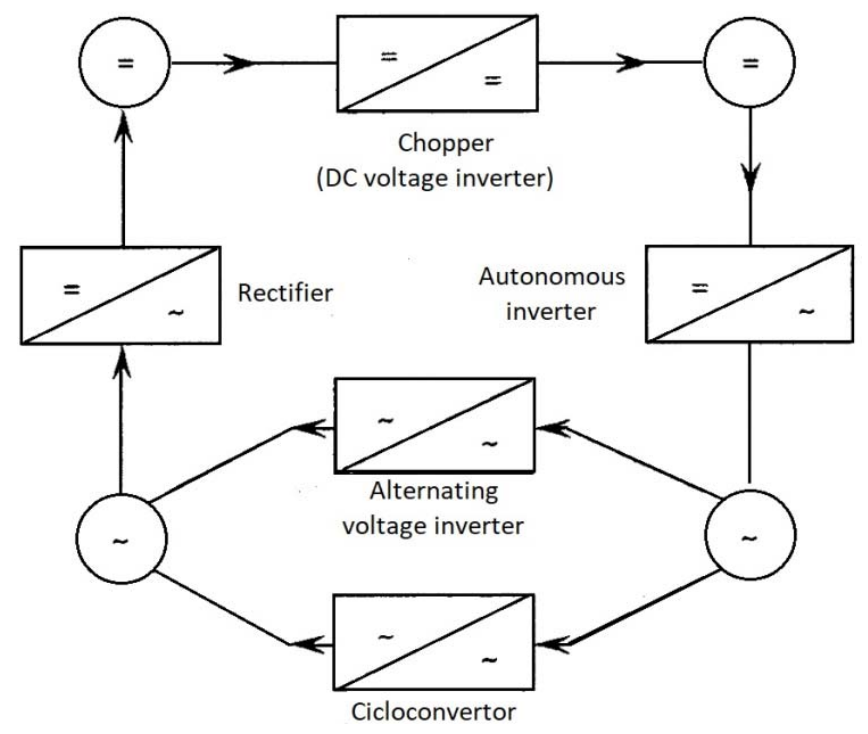

Figure 4 Energy conversion possibilities

The arrows indicate the energy flow, continuouscontinuous conversion and alternating-alternating conversion are possible with the help of converters with a more complex structure, called "intermediate phase conversion".

The forms of electricity at the input and output of the converters, respectively, are taken into account, thus four categories of static converters are distinguished, these are: rectifiers, inverters, choppers and AC converters. [8]

Generally in electrical engineering, switching means the passage of the load current from one circuit branch to another circuit branch. Energy is required to switch. In static converters, the sides that switch the load current contain semiconductor power elements, and the switching is done by closing one semiconductor element and opening another.

This criterion takes into account the way in which the energy necessary to block the semiconductor elements is ensured. There are:

1. Static converters with external or natural switching, in which the energy required to block the elements exists naturally in the circuit and comes from an external source 
(power generator or load). This category includes natural switching rectifiers, AC voltage converters, cyclo-converters, load-switching inverters (inverters that supply synchronous motors);

2. Static converters with internal or forced switching, at which the energy required for switching must be created in the structure of the converter (in the case of thyristors) or by control (in the case of fully controlled semiconductor elements). In the case of CS with thyristors and forced switching, the energy required for switching is obtained by charging the corresponding capacities. This category includes DC voltage converters, CSTF indirect inverters and forced switching rectifiers and cyclo-converters.

3. Static converters with "zero" switching are also called resonant or "soft" switching. The voltage and current through the switching elements have such a variation that they periodically go through zero. Switching is done by zero or voltage moments. Thus the energy required for switching is very small, theoretically zero. Represents a recent class of static converters. [10]

By definition, a current path contains a single switch (a single power conductor). To switch between closed and open state, a switch must have a convenient voltage applied to its terminals. This voltage is called the "switching voltage". If this voltage is available within the power part it is said that we have a converter with "natural" switching if not, it must be created and applied at certain times, in this case we have a "controlled" switching.

For normal thyristors the controlled switching is called "forced" because the blocking of such a device is done by means of an auxiliary blocking circuit, it is put into operation at a convenient time by the control circuit.

Rectifiers, AC converters and cyclo-converters are converters with "natural" switching, because the switching voltage is in the part of the force created by the alternating network. The blocking of the thyristors or diodes in the converters with natural switching is done at the natural zero crossing of the current that crosses them.

Autonomous choppers and inverters are "controlled" switching converters. The applications of static power converters belong mainly to the industrial environment because they allow the receivers to receive energy in the most convenient form, so to operate with optimal efficiency. At present, more than $60 \%$ of the energy produced is transported by static converters.

Research on the development of converters is done practically in two directions: increasing the power per unit volume and reducing the dimensions and costs.

The advances of technology based on static power converters are related to the progress of the semiconductor power devices industry, which in recent years has managed to produce devices with higher nominal characteristics and reliability.

Static converters play a key role in the electric drives used in all areas of technology. Static converters are used everywhere. They are used in the chemical industry (electrolysis, galvanization), metallurgy (steel), electrical, mechanical (welding) and also in machine tools, household appliances (speed regulation of electric machines with alternating voltage variators). [8]

\section{DESCRIPTION OF THE PROJECT}

The purpose of scientific research in this paper is to improve the operation of thermal power plants, by optimizing the movement of electromechanical actuators, which are integrated into the composition of gas valves, in terms of adaptation to the requirements of comfort, efficiency and maintenance. System optimization consists in finding new motion control solutions.

Following the operation of the thermal power plants, certain problems were found that constitute deficiencies in time of operation:

- due to voltage fluctuations on the supply network, some components of the thermal power plants (electronic board, fan, gas valve, actuators) during operation, suffer;

- the fluctuations of pressure, respectively flow, on the gas supply network, lead to problems of operation in the parameters of the thermal power plants. [1]

The research program consists in finding new solutions to control the movement of the operation of electromechanical actuators in the gas valve, used in thermal power plants, to be operated by static converters - AC voltage converter with logic gates.

\subsection{Signal analysis and circuit realization}

It analyzes and graphically represents the sinusoidal function for the signal with constant frequency $(50 \mathrm{~Hz})$ and amplitudes (A0-A4) where: A0 = $138(\mathrm{~V}) ; \mathrm{A} 1=161(\mathrm{~V})$; A2 $=184(\mathrm{~V}) ; \mathrm{A} 3=207(\mathrm{~V}) ; \mathrm{A} 4=230(\mathrm{~V})$ alternating voltage, with different values of the signal of period T. [2]

To represent two-dimensional graphs in the Matlab program, which is a development environment for numerical calculation and static analysis, we will analyze the plot function, which graphically represents a sequence of data. [14]

The analysis of the period $\mathrm{T}$ signal and the different amplitudes is represented graphically in Figure 5.

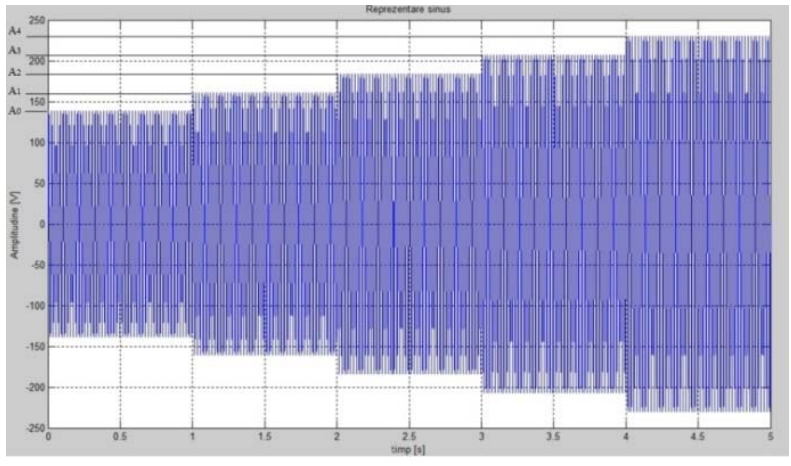

Figure 5 Graphical representation in the time domain for different amplitudes of the sinusoidal signal

The solenoid actuator is a valve with a single quickopening step, which can be implemented for motion control, a single-phase alternating voltage variable module in the AC voltage converter with logic gates (smooth start). 
Schematically, the voltage variator module can be realized as in Figure 6.

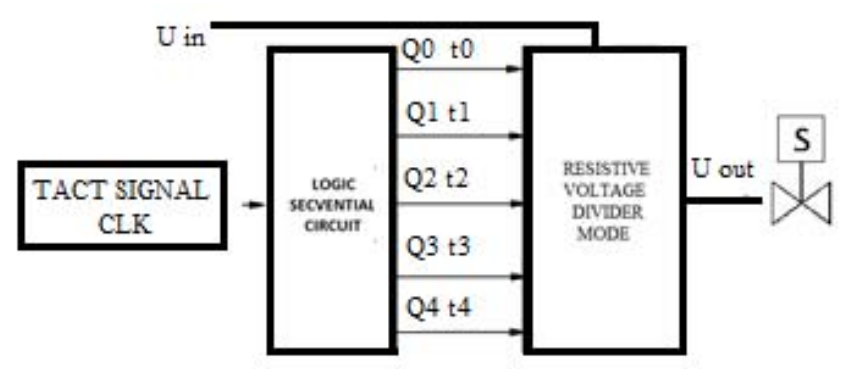

Figure 6 Voltage static convertor with logic gates where:

- TACT SIGNAL (CLK). In many digital systems it is necessary that the switching processes take place only at certain well-defined moments in time, ie they are synchronized with other signals. These synchronization signals are also called clock or clock signals (CLK).

In the presented case, an 555 time IC is used, with an oscillation time period of one second, as a signal generator. The monolithic timing circuit is an extremely stable controller capable of producing exact time or oscillation delays. In delayed mode of operation, time is precisely controlled by a single external resistor and capacitor. For stable operation as an oscillator, the free running frequency and the operating cycle are both precisely controlled with two external resistors and a capacitor. The circuit can be triggered and reset to the falling waveforms and at the output the structure can source or sink up to $200 \mathrm{~mA}$. [16]

Circuit 555 time IC has two basic operating modes: monostable mode and stable mode. In monostable mode, circuit behaves like a monostable multivibrator. It is known that a monostable multivibrator has a single steady state. Whenever a trigger pulse is applied to the input, the monostable changes from a steady state to a temporary one, a time interval remains in that state, which is set by the RC network, and then returns to its steady state.

In other words, the monostable circuit generates a single pulse of fixed duration, whenever it receives a trigger signal at the input, hence the name "one-shot". One-shot multivibrators are used to turn on or off certain external circuits or components for a certain period of time. It is also used to generate delays.

When several such multivibrators are cascaded, various forms of sequential pulses with variable filling factors can be generated. The second basic operating mode of circuit 555 is the stable mode. A stable multivibrator is actually an oscillator. The stable multivibrator generates a continuous series of rectangular off-on pulses between two voltage levels. Pulse rate and load cycle are dependent on the values of the RC network.

Figure 7 a) illustrates the circuit 555 time IC connected as a stable multivibrator. The trigger and threshold inputs (pins 2 and 6) of the two comparators are connected together to an external capacitor, C. [12]

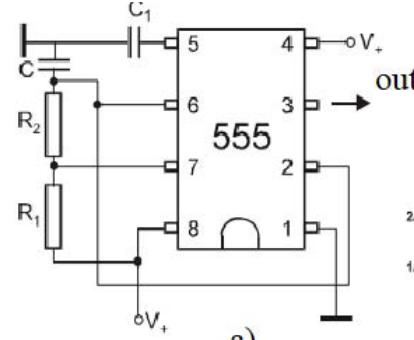

a)

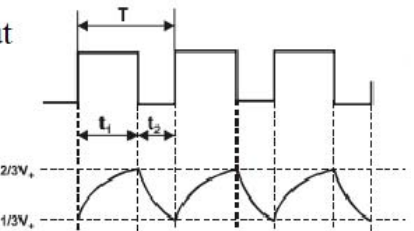

b)
Figure 7 a) time integrated circuit 555. b) time duration between pulses

The operating frequency of the stable circuit is dependent on the values of $R_{1}, R_{2}$ and $C$. This can be calculated with the formula:

$$
\mathrm{f}=1 /\left[0.693 \cdot C \cdot\left(R_{1}+2 R_{2}\right)\right]
$$

The duration of time between pulses is called the period and is usually denoted by $\mathrm{T}$.

The pulse level is at logic 1 a time tl, and at logic zero a time $\mathrm{t} 2$. The period $\mathrm{T}$ is calculated as the sum of these two time intervals: $\mathrm{T}=\mathrm{t}_{1}+\mathrm{t}_{2}$ (Figure $\left.7 \mathrm{~b}\right)$.

Period and frequency are related by the relation:

$$
\mathrm{f}=1 / \mathrm{T}
$$

The durations $\mathrm{t} 1$ and $\mathrm{t} 2$ depend on the values of $\mathrm{R} 1$ and $\mathrm{R} 2$. The ratio between the time when the output pulse is on logic $1(\mathrm{t} 1)$ and the period $\mathrm{T}$ is called the load cycle. The load cycle can be calculated with the formula:

$$
D=t_{1} / T=\left(R_{1}+R_{2}\right) /\left(R_{1}+2 R_{2}\right)
$$

The times $\mathrm{t} 1$ and $\mathrm{t} 2$ can be calculated from the formulas:

$$
\mathrm{t}_{1}=0.693 \cdot\left(R_{1}+R_{2}\right) \cdot C ; t_{2}=0.693 \cdot R_{2} C
$$

In synchronous sequential circuits, the present state of the circuit can only be changed when a timing signal called a clock or clock signal occurs. The clock signal is a series of rectangular pulses that are applied to the circuit through an additional input, called the clock signal input.

In the Figure 8, shows the elements of a clock signal (rectangular signal). [11]

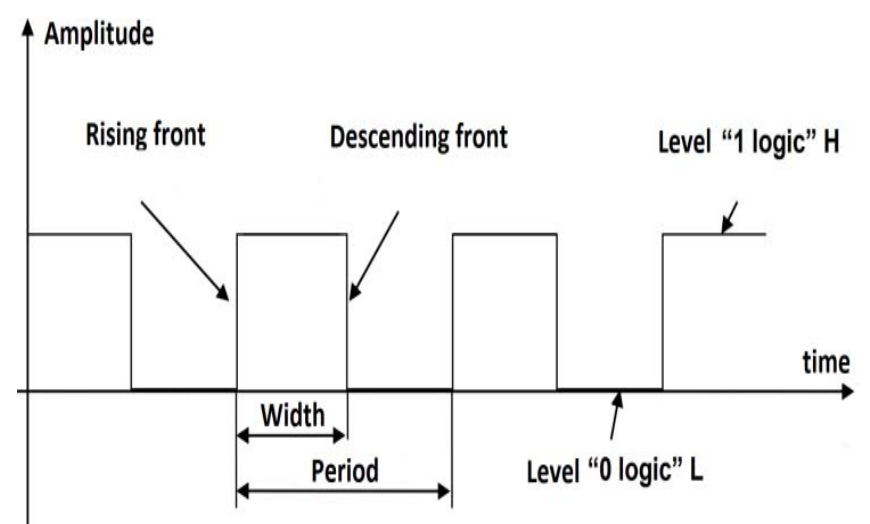

Figure 8 Elements of a clock signal (rectangular signal)

The ratio between the duration of the time and the period of the clock signal is called the filling factor. A clock signal 
can be active either on the rising edge (when the state of the circuit changes on the rising edge) or on the decreasing front (when the state of the circuit changes on the falling edge).

- LOGIC SECVENTIAL CIRCUIT is a digital circuit whose output states depend both on the combination of logic levels at inputs at a given time and on the signals applied there before, the circuit being structured both by combinational logic circuits and binary memory elements. In sequential logic circuits, the input, output, and state variables can have only two values "1logic" and "0logic" with a finite number of states.

The sequential logic circuit is a numerator, used to count the pulses applied to the input.

The counter is a device constructed of cells connected in a certain way, so that it makes it possible to determine the number of pulses arriving successively at its input, based on the analysis of the state of the tilting circuits from which its cells are retaliated.

The general structure of the counters is characterized by the cascade connection of the cells, the input terminal allowing pulses to pass into the cell with the lowest rank. The entire counting scheme is coordinated by the sequence of beats that allows the transfer of information from one cell to another. Analyzing the states of the cells after the needles have reached $\mathrm{N}$ pulses at the input terminal $\mathrm{i}$, the cells are in that state that satisfies the equality:

In which ai $(\mathrm{i}=1-\mathrm{n}-1)$

$$
\begin{gathered}
N=a_{0} 2^{0}+a_{1} 2^{1}+a_{2} 2^{2}+a_{3} 2^{3}+\cdots \\
=\sum_{i=0}^{n-1} a_{i} 2^{i}
\end{gathered}
$$

They have no data entries, so transitions are made according to a certain rule, only based on the current state. For each number in the counting range, there is a distinct state of the counter. The counting capacity is given by the number of distinct states that the counter has.

Counting can be done with the help of flip-flops and logic gates, the latter having the role of determining the correct way in which the counter changes its states in the counting process.

The number of distinct states of a counter consisting of $n$ flip-flops is $2 \mathrm{n}$, so the numerator is modulo $2 \mathrm{n}$. Each state can be associated with a binary code word of length $n$, representing the outputs of the $\mathrm{n}$ bistables for the given state of the counter. [6]

There are also counters that have less than $2 \mathrm{n}$ states, they are designed to have a different number of states than $2 \mathrm{n}$. These counters are truncated counting sequence counters. Truncating the counting sequence is obtained by forcing the counter to resume the sequence from the beginning before going through all the states. A common truncated sequence counter is the modulo 10 counter. A 10 -state counter it is called the decadic numerator.

The 4017B integrated circuit, also called the decadic counter shown in Figure 9, is used for counting applications, has the ability to activate 10 outputs sequentially in a predefined time and to reset the number or maintain it when necessary. It also has the ability to indicate the counting status using the Carry pin. This is commonly used for logical output projects. [7]

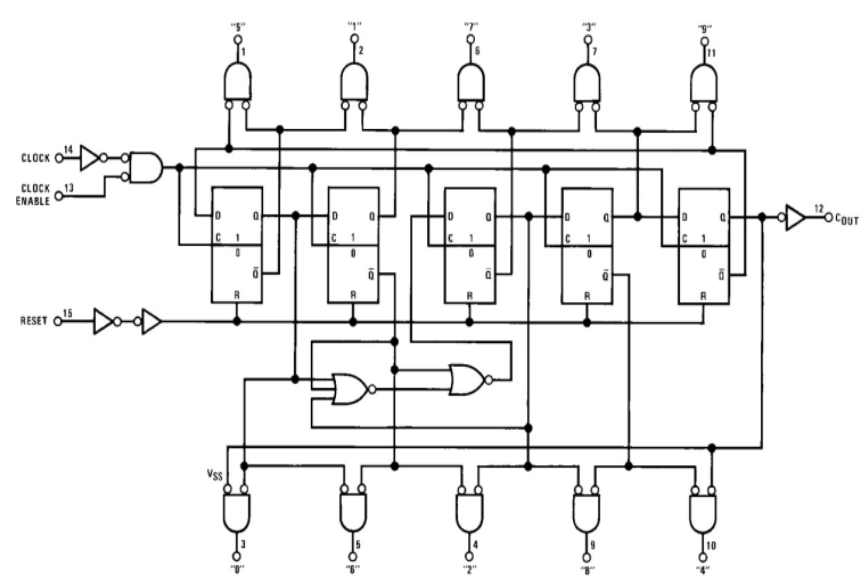

Figure 9 Logic diagram of the decadic counter

The output changes sequentially from Q0 to Q9 for each large pulse from the Clock pin, but this sequence can be interrupted by two pins. These are the Enable Clock (pin 13) and Reset (pin 15) pins. These pins are maintained ( $0 \mathrm{~V} /$ grounded) by default, but when the Enable Clock pin is raised, the countdown breaks.

For example, if the count was at pin Q3, when the clock activation pin was raised, then the count will stop at pin Q3 regardless of the pulses raised from the clock and will continue to increase only when Clock Enable is again low. Similarly, if the reset pin is raised. The number will be reset back to Q0 and will remain there until Q0 is low again.

We have another pin called the execution pin (pin 12), this pin will remain low $(0 \mathrm{~V})$ by default. But when the IC completes the number up to 10 , the pin will go up and stay high until it counts to 5 , when 5 will go down $(0 \mathrm{~V})$ and start again when it reaches 10 . IC timing diagram indicating the status of the pins output and Carry pin for each high pulse signal is shown in Figure 10. [17]

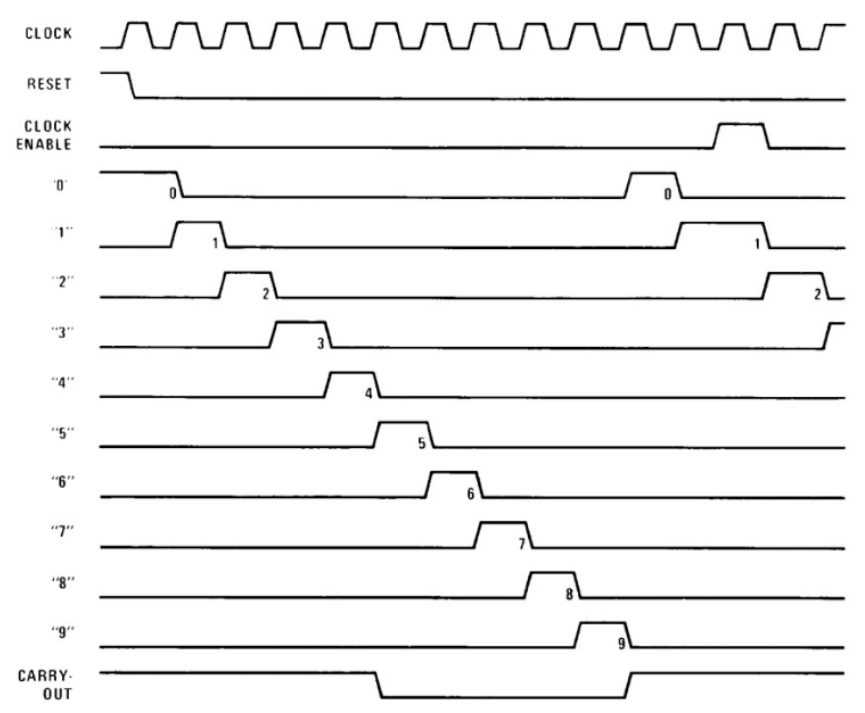

Figure 10 Diagram of time pulses of the outputs of the integrated circuit decadic counter 
- RESISTIVE VOLTAGE DIVIDER MODE is a system designed to automatically maintain a constant voltage level, can use a simple design before or can include negative feedback. It can use an electromechanical mechanism or electronic components. Depending on the design, it can be used to adjust one or more alternating or direct current voltages.

Voltage regulators or stabilizers are used to compensate for voltage fluctuations in the mains. Voltage regulators normally operate on a range of voltages, for example 130-240 $\mathrm{V}$ or 90-280 V. In electronics, a voltage divider (also known as a potential divider) is a passive linear circuit that produces an output voltage (Vout), which is a fraction of the input voltage (Vin). The voltage split is the result of the distribution of the input voltage between the divider components.

Resistive voltage dividers are commonly used to create reference voltages, or to reduce the amplitude of the voltage so that it can be measured, and can also be used as a signal attenuator at low frequencies. For direct current and relatively low frequencies, a voltage divider can be sufficiently accurate if it is made only of resistors; if a wide frequency response is required (for example in an oscilloscope), a voltage divider may have added capacitive elements to compensate for the load capacity. In the transmission of electricity, a capacitive voltage divider is used to measure high voltages [9]. The sequential outputs Q0 to Q4 of the decadic counter integrated circuit will be used to sequentially control the voltage divider.

In Figure 11 shows the resistive alternating voltage divider module as a function of time T0-4 and the sequential control Q0-Q4, for obtaining the amplitudes A0-A4, of the sinusoidal signal.

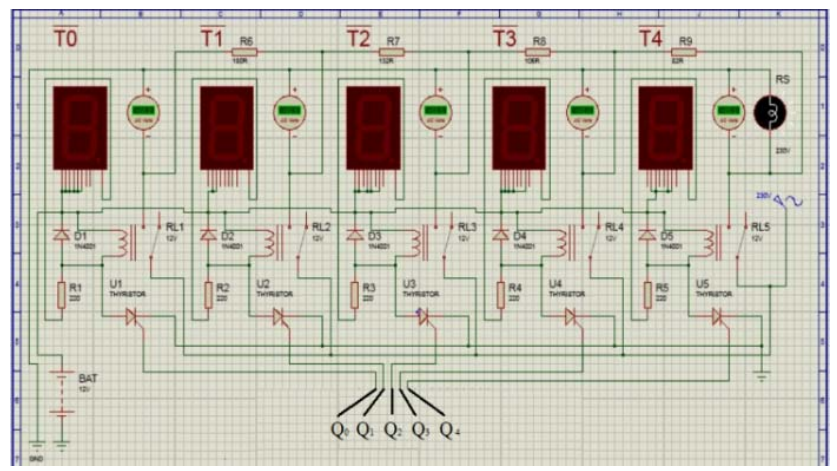

Figure 11 Voltage divider as a function of time T0-4 and sequential control Q0-4

The circuit simulation was performed in the Proteus program, the resistor group R6-R9 represents the voltage divider.

- S represents the actuator studied for the optimized control of the movement.

Sequential signal analysis was also simulated in the Proteus program and can be seen in Figure 12-16.

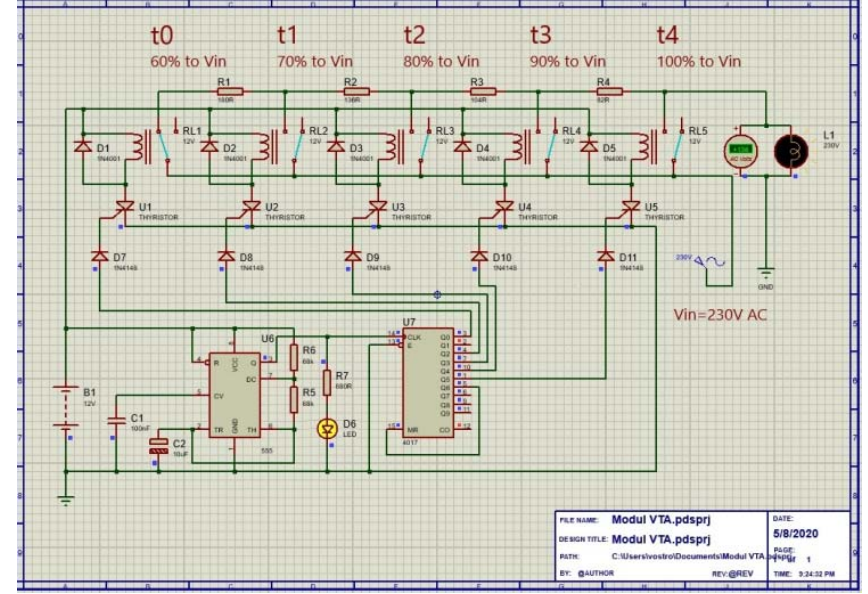

Figure 12 Sequential signal analysis in the Proteus program at time $t_{0}$

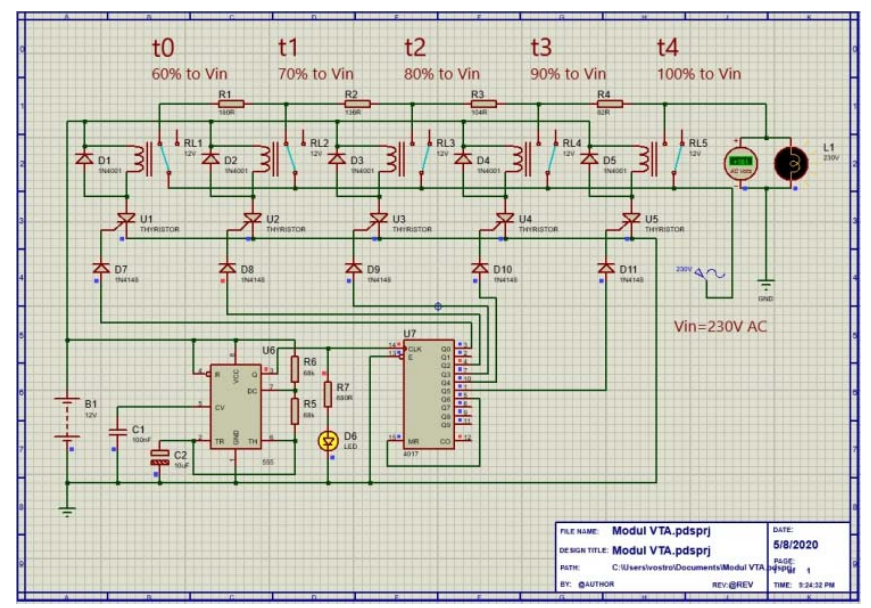

Figure 13 Sequential signal analysis in the Proteus program at time $t_{1}$

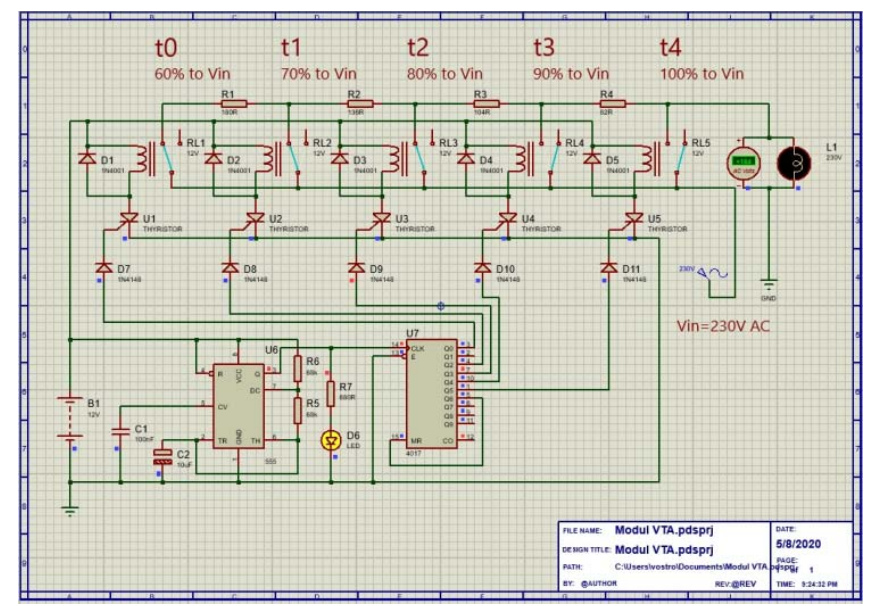

Figure 14 Sequential signal analysis in the Proteus program at time $t_{2}$ 


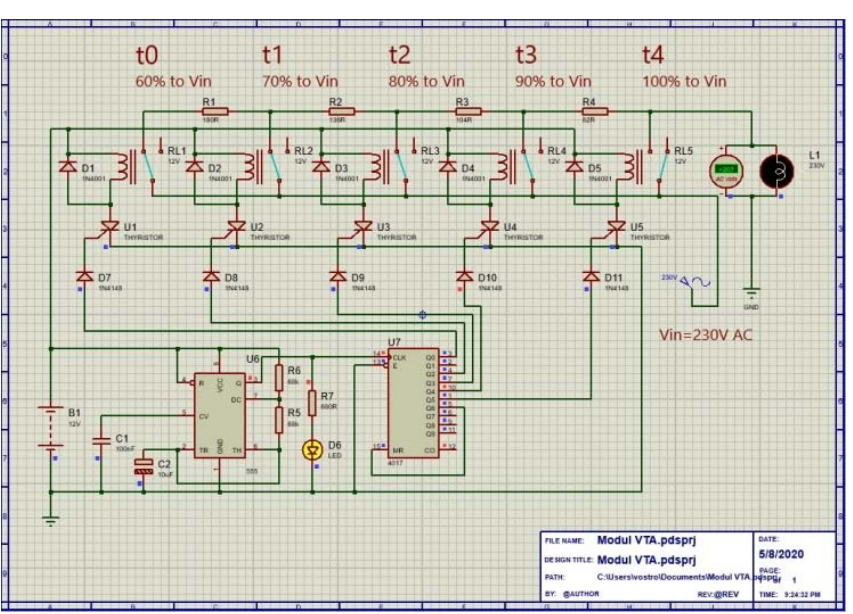

Figure 15 Sequential signal analysis in the Proteus program at time $t_{3}$

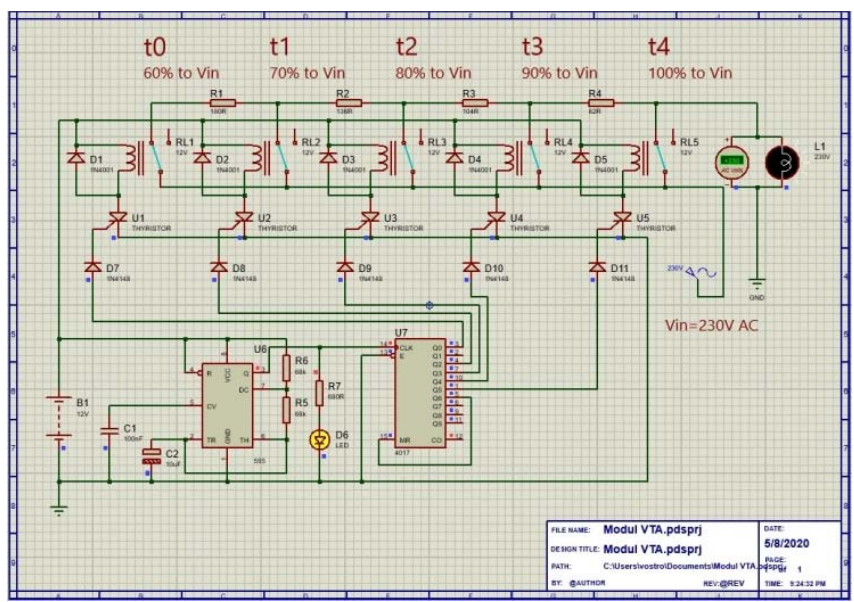

Figure 16 Sequential signal analysis in the Proteus program at time $t_{4}$

The most important common features of an easy start with single-phase alternating voltage inverter are:

-The start-ramp is the moment from which the voltage variation starts, until it reaches the maximum value. The time duration of the ramp has a value between (3-5) seconds.

-The stop-ramp is used when a smooth stop of the actuators (softstop) is needed. The stop ramp represents the time from the moment when the voltage has maximum value, until the moment when due to the decrease of the voltage, it reaches the initial value. If the time ramp is set to zero, then the stop will simply look like a direct stop.

In the case of direct mains start-up, with a start-up time of less than five seconds, a smooth start-up will be selected, depending on the power of the solenoid-type actuator, from the single-phase AC voltage module. Figure 17 shows the easy starting diagram. [13]

Establishing the values of the output voltage (V0-4 out) from the single-phase alternating voltage variable module, in the soft starter variant, through the calculated percentage values, respectively $60,70,80,90,100 \%$ of the value of the input voltage (Vin) is thus obtained an optimized control of the operation of the solenoid type actuator, which leads to a smooth operation without shocks, while reducing vibration and noise during operation.

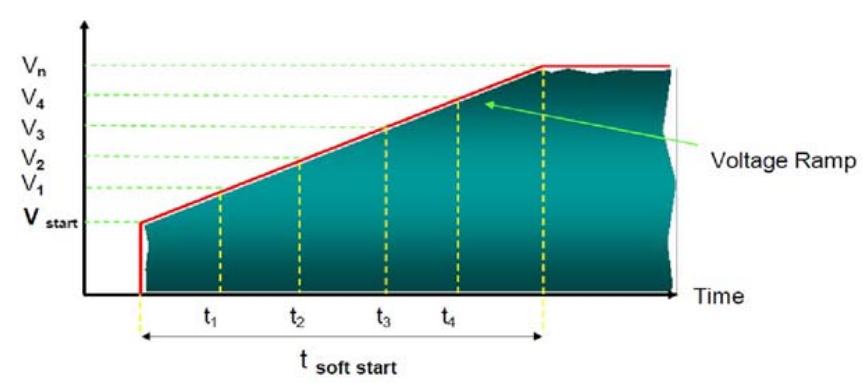

Figure 17 Voltage diagram starting ramp

\section{CONCLUSIONS}

In the present paper was presented the general state of the art on the optimized motion control technique for solenoid actuators.

By analyzing the signal made in the scientific paper [3], and the experimental realization of the research stand in the scientific paper [4], in the present paper both the simulation was performed (through the Software Proteus Design Suite developed by Labcenter Electronics Ltd and through an opensource Arduino development platform) as well as the experimental realization of a new variable voltage alternating module with logic gates.

In the presented scientific paper, by analyzing the signal applied to the actuators in the gas valve from the thermal power plants, the optimized control of the movement and regulation of the opening of the solenoid valve in time is followed.

From the above, it can be seen that the optimized motion control technique for solenoid actuators has achieved outstanding performance, but there are still potentially significant innovations. Therefore, during the successive works, new fields of research will be approached, with the intention to be based interdisciplinary with the current technical state and on the basis of experience data, of the new principles of operation.

There are various applications with implications of electromechanical actuators, which will aim to implement the time control of solenoid actuators by designing, designing and making alternating-alternating modules, also called alternating voltage variators.

The main contributions are:

- design, design and creation (realization) of a single-phase alternating voltage variable module, with active electronic components (integrated circuit, transistor, thyristor, diodes) and passive (resistor, capacitor, potentiometer, varistor)

- approach of technical solutions for motion control in applications involving solenoid actuators;

- research for the implementation of new multiparametric control strategies.

Prospects for further research is evidenced by the fact that there are many industrial applications due to the implications of diversified actuators. 


\section{REFERENCES}

[1] F. Kesucz, M. Banica, V. Nasui, "Theoretical and Experimental Aspects Regarding the Control of Actuators Used in Gas Thermal Power Plants," Scientific Buletin, Serie C, Fascicle: Mechanics, Tribology, Machine Manufacturing Technology, ISSN 1224-3264, No.XXXII, pp.27-34, 2018

[2] F. Kesucz, M. Banica, V. Nasui, “Theoretical Aspects Regarding The Optimized Control Of The Electromechanical Actuators Used In The Flow Control,"Acta Technica Napocensis - Series: Applied Mathematics, Mechanics, and Engineering, Vol. 62, Issue IV, November, pp.531-542, 2019

[3] F. Kesucz, "Theoretical and experimental aspects of signal analysis applied to solenoid-type actuators used in gas valves," Carpathian Journal of Electronic and Computer Engineering. 13/1 (2020) 13-22. DOI:10.2478/cjece-2020-0003.

[4] F. Kesucz, "Experimental modeling of the optimized motion control of electromechanical actuators used in thermal power plants," Carpathian Journal of Electronic and Computer Engineering. 13/1 (2020) 30-40. DOI:10.2478/cjece-2020-0005.

[5] V. Năsui, "Bazele cercetarii experimentale," Editura Universitatii de Nord - Baia Mare, pp.3-4, 2000.
[6] Pop, E, Stoica, V., Principii si metode de măsurare numerică, Editura Facla, Timișoara, pp.112-117, 1977

[7] ***https://components101.com/cd4017-pinout-datasheet

[8] $* * *$ https://electrokits.ro/carti-electronica-c-4/carti-electronica-ro-c4_5/electronica-de-putere-convertoare-statice-p-15.html

[9] ***https://electronic-club.com/divizor-de-tensiune/

[10] ***http://elth.ucv.ro/fisiere/problem $\% 20$ studentesti/Cursuri/Converto are $\% 20$ statice/Convertoare $\% 20$ statice\%20-\%20IEC.pdf

[11] $* * *$ https://eprofu.ro/docs/electronica/digitala/cap6.pdf

[12] ***http://phys.ubbcluj.ro/ anghels/teaching/SIS_hide $\% 5 \mathrm{D} /$ diverse_m ateriale/temporizatorul_555.pdf

[13] ***http://site.ieee.org/houston/files/2016/09/IEEE-HOU-Sec20160922-Motor-Control-Seminar2.pdf

[14] ***http://web.ulbsibiu.ro/catalina.neghina/Resurse/carti/indrumarPN S.pdf

[15] ***https://www.atlantic-comfort.com/Atlantic-Brand/ERP-Energyrelated-Products-Directive

[16] ***http://www.555-timer-circuits.com/datasheets/ne555.pdf

[17] $* * *$ http://www.ti.com/lit/ds/schs027c/schs027c.pdf?ts $=15887563970$ 69 